\title{
Utilidad de la ecocardiografía en la detección de la insuficiencia cardiaca en un adulto joven con síndrome de origen anómalo de la arteria coronaria izquierda del tronco de la arteria pulmonar y válvula mitral asimétrica similar al paracaídas
}

\author{
Luis Cordero ${ }^{a, c}$, Jaime Rodríguez ${ }^{a, c}$, José Zuluaga ${ }^{a, c}$, Fernán Mendoza ${ }^{b, *}$ \\ y Osmar Pérez ${ }^{b}$ \\ a Departamento de Métodos no invasivos y Ecocardiografía, Fundación Clínica Shaio, Bogotá, Colombia \\ b Departamento de Cardiología Clínica, Fundación Clínica Shaio, Bogotá, Colombia \\ c Universidad del Rosario, Bogotá, Colombia
}

Recibido el 18 de enero de 2017; aceptado el 24 de abril de 2017

Disponible en Internet el 31 de agosto de 2017

\section{PALABRAS CLAVE}

Insuficiencia

cardiaca;

Insuficiencia mitral;

Coronaria izquierda;

Cardiopatía congénita

\section{KEYWORDS}

Heart failure;

Mitral insufficiency;

Left coronary artery;

Congenital

heartdisease

\begin{abstract}
Resumen
Objetivo: describir un caso de un paciente joven con insuficiencia cardiaca, secundaria a dos malformaciones cardiacas infrecuentes, síndrome de ALCAPA y válvula mitral asimétrica, parecida al paracaídas, resaltando la utilidad de la ecocardiografía. Métodos: se analiza el caso a la luz de la literatura médica.

Conclusiones: El origen anómalo de la arteria coronaria izquierda del tronco de la arteria pulmonar y la válvula mitral asimétrica parecida al paracaídas, son malformaciones raras, asociadas a insuficiencia mitral severa e insuficiencia cardiaca. No se encontraron reportes en la literatura acerca de la coexistencia de las dos patologías en un paciente.

(c) 2017 Sociedad Colombiana de Cardiología y Cirugía Cardiovascular. Publicado por Elsevier España, S.L.U. Este es un artículo Open Access bajo la licencia CC BY-NC-ND (http:// creativecommons.org/licenses/by-nc-nd/4.0/).
\end{abstract}

Use of echocardiography in the detection of heart failure in a young adult with anomalous left coronary artery from the pulmonary artery syndrome andparachute-like asymmetric mitral valve

Abstract

Objective: To describe a case of a young adult with heart failure, secondary to two rare cardiac malformations, anomalous left coronary artery from the pulmonary artery

\footnotetext{
* Autor para correspondencia.

Correos electrónicos: fernan.mendozafmb@gmail.com, fernan.mendoza@shaio.org (F. Mendoza).
} 
(ALCAPA) syndrome and parachute-like asymmetric mitral valve, highlighting the use of echocardiography.

Material and methods: A case is analysed along with a search in the medical literature.

Conclusions: The anomalous origin of left coronary artery from the pulmonary artery and parachute-like asymmetric mitral valve are rare malformations associated with severe mitral insufficiency and heart failure. No reports were found in the literature as regards the existence of these two diseases in a patient.

(c) 2017 Sociedad Colombiana de Cardiología y Cirugía Cardiovascular. Published by Elsevier España, S.L.U. This is an open access article under the CC BY-NC-ND license (http:// creativecommons.org/licenses/by-nc-nd/4.0/).

\section{Introducción}

De acuerdo con la $32^{\mathrm{a}}$ conferencia de Bethesda en el 2000, hay aproximadamente 2.800 adultos con cardiopatías congénitas por cada millón de habitantes y más de la mitad de ellos tienen defectos de moderada a alta complejidad $^{1}$. De los pacientes con cardiopatías congénitas detectados previamente en la infancia, coexiste un grupo cuyo diagnóstico se hace en la edad adulta como hallazgo incidental, por enfermedad cardiaca concomitante $y$, con mayor frecuencia, por presencia de síntomas que llevan al descubrimiento de la lesión. Las cardiopatías congénitas en la edad adulta se asocian con insuficiencia cardiaca, arritmias, complicaciones vasculares y muerte súbita ${ }^{2}$. Cuando se efectúa un estudio ecocardiográfico a un paciente adulto con signos y síntomas de insuficiencia cardíaca, se deben tener presentes las formas raras de cardiopatías congénitas $^{3}$.

El origen anómalo de la arteria coronaria izquierda del tronco de la arteria pulmonar (ALCAPA, por su sigla en inglés "Anomalous Left Coronary Artery from the Pulmonary Artery"), también conocido como síndrome Bland-White-Garland, tiene una incidencia de 1 en 300.000 nacidos vivos, representa del $0,25 \%$ al $0,5 \%$ de todas las cardiopatías congénitas. Es una anomalía rara de las arterias coronarias $^{4}$. Hay dos variedades: la infantil que se asocia a una mortalidad del $90 \%$ en el primer año de vida, y la del adulto que corresponde al $10 \%$ a un 15\%. Aquellos que sobreviven lo hacen gracias a una arteria coronaria derecha dominante y a una extensa red de colaterales intercoronarias, que llenan el sistema coronario izquierdo ${ }^{5}$. Esta anomalía se presenta en forma aislada en la mayoría de casos, pero en un $5 \%$ se asocia a otras malformaciones congénitas como tetralogía de Fallot, comunicación interventricular, transposición de grandes arterias, coartación de aorta y conducto arterioso permeable ${ }^{6}$.

De otra parte, las malformaciones congénitas de la válvula mitral, representan del $0,21 \%$ al $0,42 \%$ de todas las cardiopatías congénitas ${ }^{3,7}$. La válvula mitral en paracaídas tiene una prevalencia estimada del $0,1 \%$ al 0,2\%. Particularmente, la incidencia en adultos es muy baja y pocos casos son reportados ${ }^{8}$. La válvula mitral en paracaídas consiste en la inserción de las cuerdas tendinosas de ambos velos de la válvula mitral en un único músculo papilar. Se denomina "verdadera"' si solo existe un músculo papilar. Por otro lado, en la válvula mitral similar al paracaídas asimétrica (parachute-like asymmetric mitral valve), las cuerdas tendinosas están distribuidas en forma desigual entre los dos músculos papilares identificados. Usualmente, el músculo papilar dominante es el normal (más común el posteromedial) y el otro es hipoplásico ${ }^{8,9}$. Los varones son afectados con mayor frecuencia y se encuentran otras anomalías congénitas cardiacas en más del $99 \%$ de los $\operatorname{casos}^{10}$, aunque en la adultez es más común encontrarla en forma aislada ${ }^{11}$.

Se describe un caso de un paciente adulto joven, con insuficiencia cardiaca y cardiopatía dilatada en quien se diagnosticaron síndrome de ALCAPA y válvula mitral asimétrica similar al paracaídas, mediante ecocardiografía transtorácica y cateterismo cardiaco. En la revisión de la literatura no se encontraron otros casos que reportaran esta coincidencia.

\section{Caso}

Paciente varón, de 30 años de edad, con diagnóstico de insuficiencia mitral severa desde la infancia, de etiología sin esclarecer, insuficiencia cardiaca congestiva, síncope y palpitaciones, todo lo anterior asociado a cardiopatía dilatada; era además, portador de cardiodesfibrilador automático implantable como prevención primaria. Ingresó a la institución con deterioro de la clase funcional hasta clase IV de la New York Heart Association, ortopnea y disnea paroxística nocturna junto con edemas periféricos. Al examen clínico de ingreso se identificó soplo holosistólico en foco mitral con disminución del primer ruido e irradiación a la axila grado $3 / 6$, estertores crepitantes en ambas bases pulmonares, ascitis y edemas periféricos. En la radiografía de tórax se documentó cardiomegalia, derrame pleural bilateral y signos de congestión pulmonar. Fue hospitalizado para compensación y ampliación de estudios. Se realizó ecocardiograma transtorácico en el que evidenció insuficiencia mitral severa de origen mixto, con predominio del componente funcional por la presencia de tethering (o tracción en español) de ambas valvas y dilatación del anillo, que midió $55,0 \mathrm{~mm}$, compromiso del aparato subvalvular por presencia de músculo papilar posteromedial normal y músculo papilar anterolateral hipoplásico, que genera cuerdas tendinosas a su propia valva, configurando una válvula mitral similar al paracaídas (parachute like asymmetric mitral valve) 


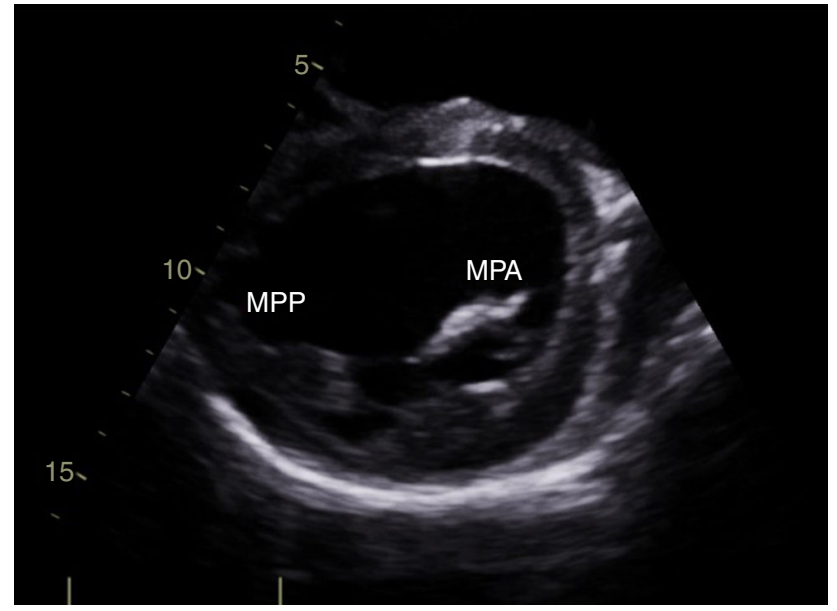

Figura 1 Imagen obtenida mediante ecocardiograma transtorácico. Vista paraesternal eje corto. MPA: músculo papilar anterolateral hipoplásico. MPP: músculo papilar posteromedial.

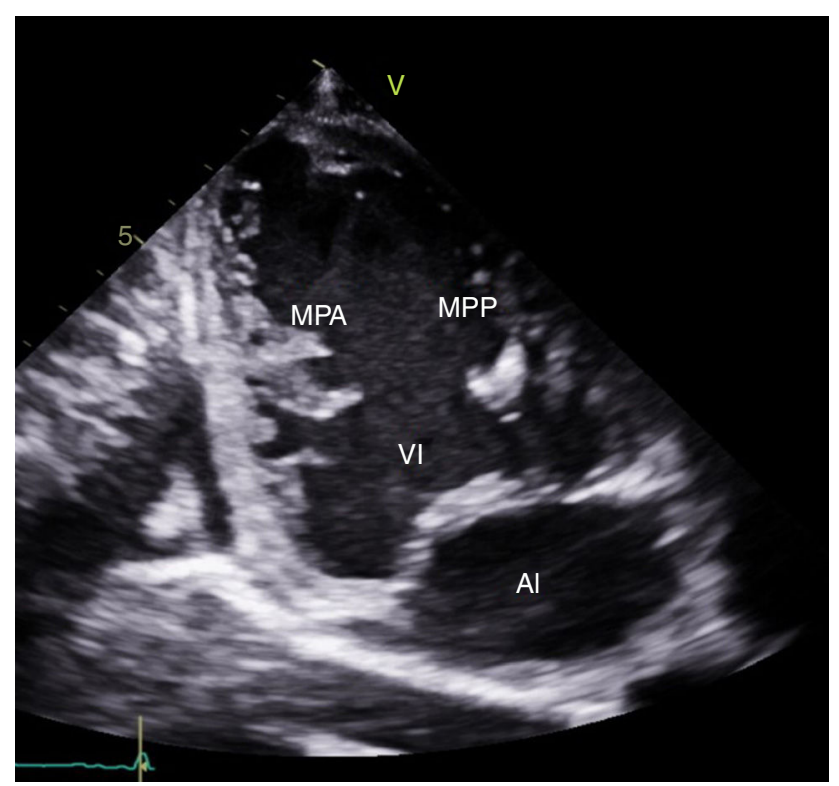

Figura 2 Imagen obtenida mediante ecocardiograma transtorácico. Vista apical dos cámaras. MPA: músculo papilar anterolateral hipoplásico. MPP: músculo papilar posteromedial. vI: ventrículo izquierdo. Al: aurícula izquierda.

(figs. 1-3). Cardiopatía dilatada de probable origen mixto (isquémico y valvular), compromiso moderado de la función sistólica del ventrículo izquierdo, fracción de eyección del 35\%, sin evidencia de alteraciones segmentarias de la contractilidad. Origen apropiado de la arteria coronaria derecha, que se observa dilatada. No se visualizó el origen de la arteria coronaria izquierda en su seno de Valsalva. En contraposición, se observó arteria pulmonar dilatada en el tronco y sus ramas de donde se origina la arteria coronaria izquierda (fig. 4). Dilatación auricular izquierda. Cavidades derechas dilatadas, con función sistólica preservada del ventrículo derecho. Electrodo de estimulación eléctrica bien posicionado.

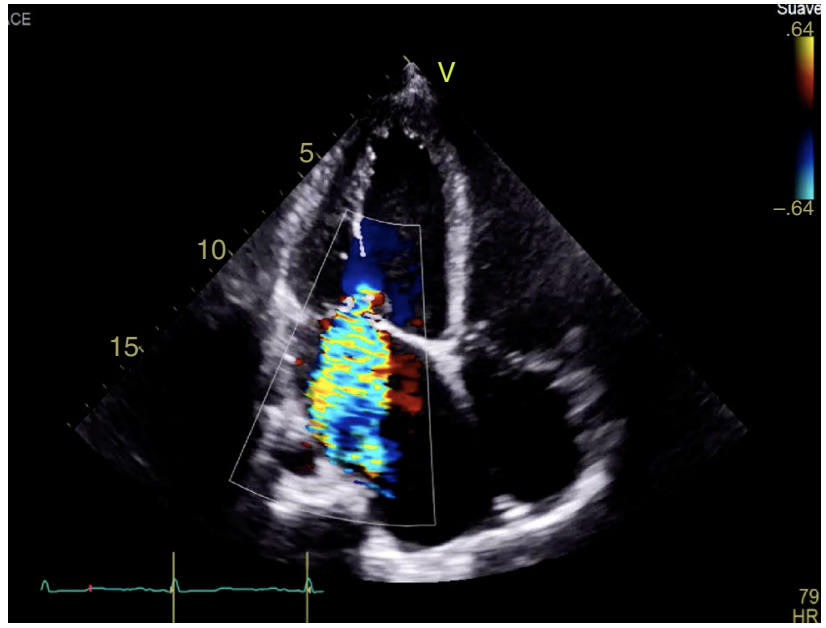

Figura 3 Insuficiencia mitral severa con doble mecanismo: el primero por la asimetría generada por el músculo papilar anterolateral hipotrófico y el segundo, insuficiencia mitral funcional por la dilatación de cavidades izquierdas, producto de la cardiopatía isquémica asociada al síndrome de ALCAPA.

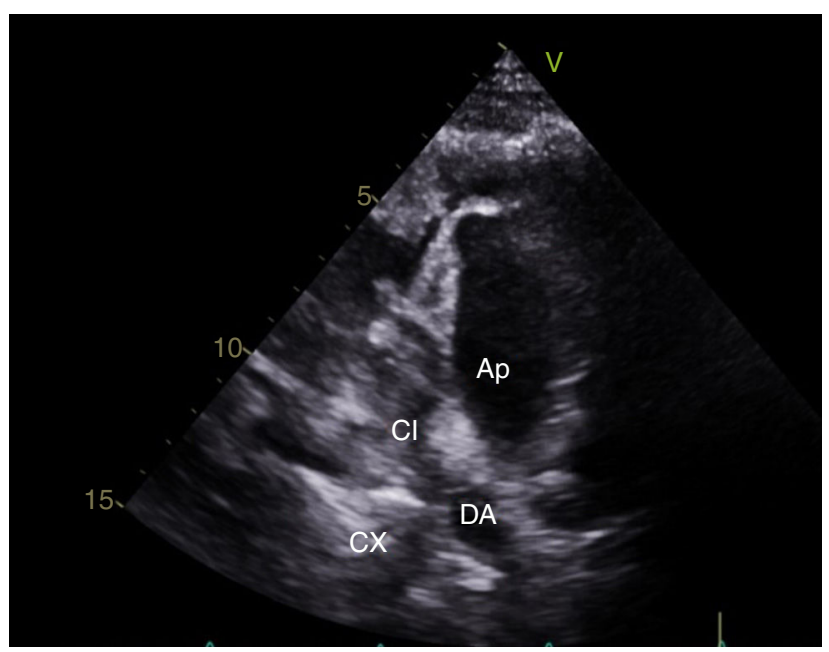

Figura 4 Imagen obtenida mediante ecocardiograma transtorácico. Eje corto de la arteria pulmonar. AP: arteria pulmonar. $\mathrm{Cl}$ : arteria coronaria izquierda. DA: arteria descendente anterior. CX: arteria circunfleja.

La ausencia de ostium coronario izquierdo en el seno de valsalva correspondiente y su origen en la arteria pulmonar, se detectaron tanto por ecocardiografía como en el cateterismo (figs. 5 y 6). Se inició terapia farmacológica completa para insuficiencia cardiaca (inhibidores de la enzima convertidora de la angiotensina, espironolactona, carvedilol, furosemida), observándose mejoría transitoria de la clase funcional. En la actualidad, está en estudios para trasplante cardíaco o asistencia ventricular. Los últimos estudios documentaron hipertensión pulmonar.

\section{Discusión}

La ecocardiografía transtorácica es la modalidad de imagen de primera línea en el adulto con cardiopatías congénitas, 


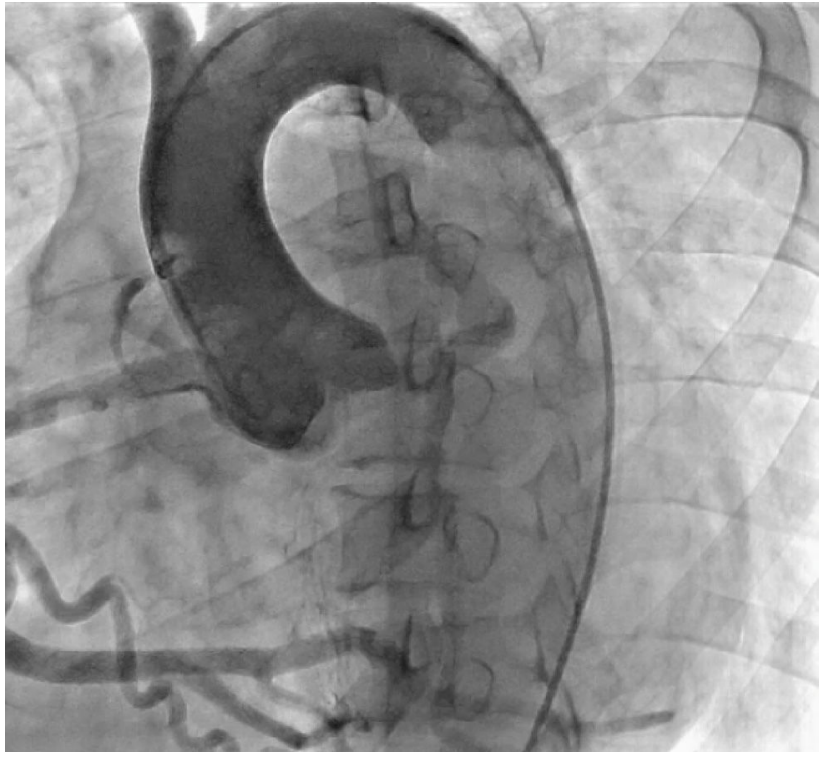

Figura 5 Nacimiento normal de la coronaria derecha a partir de la aorta; no se observa el nacimiento de la coronaria izquierda a partir de ésta.

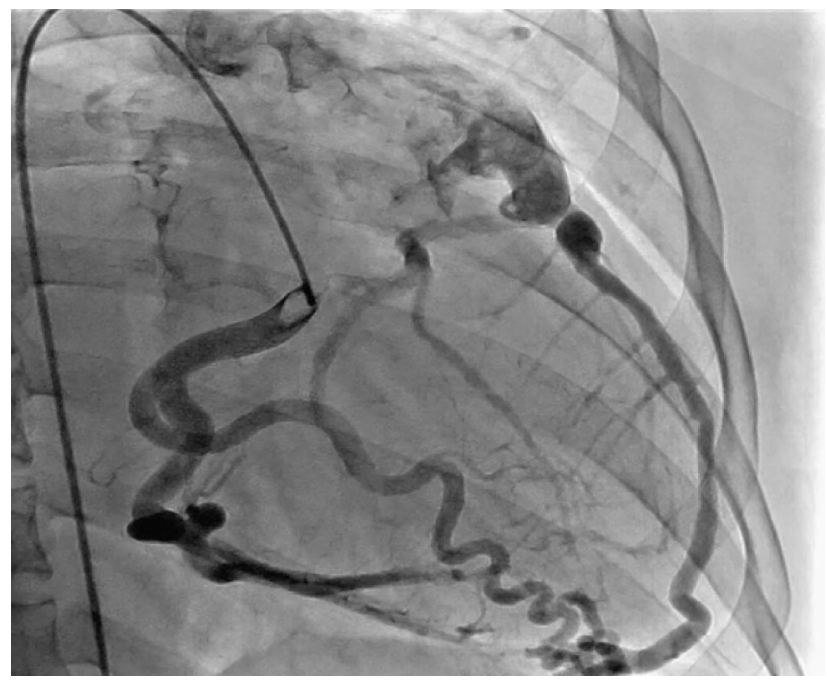

Figura 6 Nacimiento anómalo de la arteria coronaria izquierda a partir del tronco de la arteria pulmonar, configurando el síndrome de ALCAPA.

principalmente aquellos con enfermedad valvular. Se pueden evaluar la anatomía cardiaca (orientación y conexiones veno-auricular, aurículo-ventricular y ventrículo-arterial), la morfología de las estructuras cardiacas, la función ventricular, la valoración de las válvulas, la presencia de cortocircuitos y la función hemodinámica. Permite además visualizar de manera detallada las estructuras móviles intracardiacas así sean pequeñas y delgadas, y anormalidades de las valvas, los tabiques y las vegetaciones si las hay ${ }^{12}$.

El paciente del caso fue admitido a la institución por deterioro de la clase funcional y signos claros de insuficiencia cardíaca. Al realizar estudios para determinar la etiología, se practicó un ecocardiograma transtorácico encontrando una válvula mitral similar al paracaídas asimétrica y posteriormente se diagnosticó el origen anómalo de la coronaria izquierda en la arteria pulmonar por cateterismo y ecocardiografía; ambas malformaciones tienen baja incidencia, especialmente en la edad adulta. No se encontraron otros reportes en la literatura donde coincidieran las dos alteraciones.

La insuficiencia cardiaca es una complicación multifactorial frecuente de los pacientes adultos con cardiopatías congénitas, asociada a aumento en la morbimortalidad e ingresos hospitalarios. Es la causa más frecuente de muerte en este grupo de pacientes, y ocasiona aproximadamente el $26 \%$ de los fallecimientos ${ }^{13}$. La admisión hospitalaria por insuficiencia cardiaca aumenta hasta cinco veces la mortalidad de los pacientes adultos con cardiopatías congénitas en comparación con los no admitidos. De modo que estos pacientes son especialmente vulnerables y ameritan un seguimiento estrecho, una evaluación anatómica exhaustiva para detectar alteraciones estructurales potencialmente reparables e intervenciones médicas tempranas ${ }^{14}$. El sexo masculino es un factor adicional que también aumenta la mortalidad en pacientes con cardiopatías congénitas admitidos por insuficiencia cardiaca ${ }^{14}$.

La válvula mitral en paracaídas recibe este nombre por la similitud de la válvula deformada con un paracaídas, descrita por Shone et al. en 1963. Las valvas se asemejan al velo del paracaídas, las cuerdas tendinosas a las cuerdas del paracaídas y el músculo papilar al arnés ${ }^{15}$. Las cuerdas tendinosas con frecuencia son poco desarrolladas, de tal forma que afectan la movilidad de la válvula mitral debido a la reducción marcada de los espacios intercordales. La alteración en la localización del músculo papilar y en la distribución de las cuerdas se acompaña generalmente de una reducción en el tamaño del orificio mitral durante la diástole, que obstruye el flujo de sangre desde la aurícula hasta el ventrículo izquierdo y afecta la función de la válvula mitral; además, puede producir remodelamiento adaptativo del ventrículo izquierdo. La reducción del tamaño del orificio mitral tiene una fuerte correlación con el grado de asimetría de los músculos papilares ${ }^{16}$. La presentación clínica de la válvula en paracaídas es variada; existen incluso casos asintomáticos, aunque poco frecuentes. La mayoría de casos se acompaña de otras malformaciones obstructivas del corazón izquierdo, pudiendo constituir formas completas e incompletas del complejo de Shone ${ }^{8}$. La mayoría de diagnósticos se realiza mediante ecocardiografía, siendo aconsejable revisar cuidadosamente la válvula mitral ante la presencia de cualquier malformación cardiaca para descartar un defecto en este lugar ${ }^{17}$. En este caso se trata de una variedad de válvula mitral en paracaídas "no verdadera", ya que existen dos músculos papilares; uno hipoplásico, el anterolateral, con distribución de las cuerdas entre ambos.

Respecto al síndrome de ALCAPA, su fisiopatología se desarrolla en cuatro fases. En la etapa fetal y neonatal, la alta presión pulmonar y la hemoglobina fetal, proporcionan perfusión y oxigenación adecuadas al miocardio. Pero, en la infancia temprana en una segunda fase, la caída de la presión pulmonar y en los niveles de oxígeno sumada al aumento en el trabajo del ventrículo izquierdo, produce isquemia que lleva a una descompensación más notoria en situaciones en las que aumenta la demanda de oxígeno miocárdico, como la alimentación o el llanto. 
La isquemia miocárdica crónica altera la función tanto del miocardio como del aparato valvular mitral, conduciendo a un insuficiencia cardiaca. Si esta fase es tolerada, ocurren cambios compensatorios y remodelamiento del miocardio. La tercera fase corresponde a los pacientes que sobreviven hasta la edad adulta debido al desarrollo de colaterales intercoronarias y a una gran arteria coronaria derecha dominante que suple a la izquierda. Esto ocasiona un flujo invertido desde la arteria coronaria izquierda hacia la pulmonar. Durante la cuarta fase, que se aprecia también en adultos, se puede producir una desviación de la sangre por la excesiva red de colaterales, desde la coronaria derecha hacia la izquierda y la arteria pulmonar (robo coronario).

Puede desarrollarse una adaptación nueva con estenosis ostial de la coronaria izquierda o restricción de su apertura hacia la pulmonar; se describen vasos colaterales de la arteria bronquial hacia el miocardio. Los pacientes adultos con ALCAPA se adaptan a la isquemia crónica, a la perfusión pobre y a un cortocircuito arterial-venoso. La falta de estas adaptaciones contribuye a la morbimortalidad.

La isquemia crónica produce disfunción sistólica ventricular izquierda y dilatación del ventrículo ${ }^{18}$. La zona más afectada es la pared anterolateral de ventrículo izquierdo y el músculo papilar mitral homónimo, dos de los elementos en los que se basa la forma de presentación.

La manifestación clínica de ALCAPA es variable; los pacientes pueden ser asintomáticos o presentar disnea, angina, fatiga y palpitaciones. Desarrollan insuficiencia mitral, insuficiencia cardiaca progresiva, miocardiopatía isquémica, fibroelastosis endomiocárdica difusa del ventrículo izquierdo, hipertensión pulmonar y arritmias ventriculares malignas. Como se mencionó, el $80 \%$ al $90 \%$ de los pacientes presentan arritmias que pueden llevar a muerte súbita. Este riesgo existe hasta en pacientes asintomáticos, ocurre generalmente en la tercera década de la vida y clásicamente es precipitado por el ejercicio.

Pocos toleran la alteración prolongada de la perfusión coronaria $^{10,19}$. Son síntomas de alarma sugestivos de anomalías de las coronarias, aquellos asociados al ejercicio, como dolor torácico, vértigo y síncope, específicamente durante o justo al terminar el ejercicio ${ }^{20}$.

Los pacientes con ALCAPA pueden tener además disfunción grave aguda de la válvula mitral y del músculo papilar ${ }^{18}$. La insuficiencia mitral es multifactorial y puede explicarse por la dilatación del anillo mitral (secundaria a la dilatación ventricular izquierda), isquemia, infarto, fibrosis del músculo papilar y alteración en la contractilidad de la pared libre del ventrículo izquierdo9. En este caso, se cree que puede tratarse de dos alteraciones: una válvula mitral en paracaídas "no verdadera" y el síndrome de ALCAPA. Otra posibilidad es que el síndrome de ALCAPA produjo los cambios sobre el músculo papilar anterolateral y dilatación del anillo mitral ocasionando insuficiencia mitral severa.

El síndrome de ALCAPA puede explicarse por varias teorías: una disposición anómala del septum aorto-pulmonar y un defecto en la separación para generar las arterias aorta y pulmonar. La segunda consiste en que el primordio que origina la coronaria izquierda, se localiza de manera incorrecta en el territorio de la arteria pulmonar y la tercera es que la aorta y la arteria pulmonar tengan la capacidad para generar los brotes de donde se generan las arterias coronarias.

La ecocardiografía transtorácica es el estudio de primera línea y es una herramienta de gran ayuda para el diagnóstico de ALCAPA, ya que permite visualizar el origen anómalo de la coronaria izquierda, la coronaria derecha dilatada, el llenado retrógrado de la coronaria afectada, el flujo diastólico anormal en la arteria pulmonar y las señales de flujo anormales septales o epicárdicas provenientes de las colaterales, además de las alteraciones sobre la válvula mitral.

En pacientes con síntomas cardiacos es importante excluir la presencia de anomalías de las coronarias, especialmente se debería tener un alto índice de sospecha de ALCAPA en lactantes con cardiopatía dilatada e insuficiencia mitral ${ }^{18,19}$. En la radiografía de tórax se puede encontrar cardiomegalia o edema pulmonar, como en el paciente del caso $^{21}$. El manejo de los pacientes sintomáticos con disfunción ventricular izquierda progresiva, es un reto. El tratamiento se basa mayormente en las guías de manejo del adulto con cardiopatías congénitas y en la extrapolación de la evidencia en el manejo de la insuficiencia cardiaca ${ }^{18}$.

En este caso se encuentran dos malformaciones cardiacas que producen insuficiencia mitral severa, remodelamiento ventricular e insuficiencia cardiaca, que explican el cuadro clínico, los signos y síntomas. Es sorprendente que dada la alta mortalidad del ALCAPA en forma aislada, este paciente haya llegado a la edad adulta, aún en presencia de una segunda malformación grave.

Una vez hecho el dignóstico en lactantes, se llevan a cirugía de urgencia. En los pacientes que llegan a la vida adulta la decisión es difícil.

Este caso fue valorado por una junta médico-quirúrgica que decidió que era de alto riesgo para una corrección quirúrgica; por tanto, se propuso iniciar estudios para trasplante cardiaco y/o asistencia ventricular como puente o terapia de destino.

\section{Conclusiones}

La evaluación del adulto con enfermedad cardiaca establecida o síntomas sugestivos de origen cardiaco, debe incluir un estudio ecocardiográfico en el que se descarten en forma precisa y exhaustiva malformaciones congénitas. Cada vez se diagnostican cardiopatías congénitas en el adulto considerándose un reto para el cardiólogo. En esta revisión se presenta el caso de un paciente con síndrome de ALCAPA y una anormalidad de la válvula mitral.

\section{Responsabilidades éticas}

Protección de personas y animales. Los autores declaran que para esta investigación no se han realizado experimentos en seres humanos ni en animales.

Confidencialidad de los datos. Los autores declaran que han seguido los protocolos de su centro de trabajo sobre la publicación de datos de pacientes. 
Derecho a la privacidad y consentimiento informado. Los autores declaran que en este artículo no aparecen datos de pacientes.

\section{Conflictos de interés}

Ninguno.

\section{Agradecimientos}

\section{A la Fundación Clínica Shaio.}

\section{Bibliografía}

1. Baumgartner H, Bonhoeffer P, De Groot NM, de Haan F, Deanfield JE, Galie N, et al. Guía de Práctica Clínica de la ESC para el manejo de cardiopatías congénitas en el adulto (nueva versión 2010). Rev Esp Cardiol. 2010;63:e1-59, 1484.

2. Bhatt AB, Foster E, Kuehl K, Alpert J, Brabeck S, Crumb S, et al. Congenital heart disease in the older adult: a scientific statement from the American Heart Association. Circulation. 2015;131:1884-931.

3. Motoda H, Murata M, Iwanaga S, Matsushita K, Nakamizo $H$, Wakino S, et al. Parachute mitral valve incidentally diagnosed in an adult patient with hypertension. J Echocardiogr. 2010;8:28-9.

4. Lorenzana JRP, Rodríguez JAL, Garrido EHN, Perezgrovas MAC, Martínez JAS, Alanís EA, et al. Origen anómalo de la arteria coronaria izquierda del tronco de la arteria pulmonar de un paciente adulto. Rev Esp Méd Quir. 2012;17:51-5.

5. Mungan U, Ozeke O, Mavioglu L, Sarisahin M, Ertan C, Demir $A D$, et al. Adult type anomalous left coronary artery arising from the pulmonary artery (ALCAPA): complementary role of multimodality cardiac imaging. Herz. 2014;39:1010-2.

6. Safaa AM, Du LL, Batra R, Essack N. A rare case of adult type ALCAPA syndrome: presentation, diagnosis and management. Heart Lung Circ. 2013;22:444-6.

7. Feng TY, Li ZA, He YH, Han JC, Luan SR, Wang LL. Parachute mitral valve accompanied by bicuspid aortic valve on threedimensional transesophageal echocardiography. Kaohsiung J Med Sci. 2012;28:506-8.

8. Marino BS, Kruge LE, Cho CJ, Tomlinson RS, Shera D, Weinberg PM, et al. Parachute mitral valve: morphologic descriptors, associated lesions, and outcomes after biventricular repair. J Thorac Cardiovasc Surg. 2009;137:385-93, e4.

9. Gunturiz-Beltrán C, Rodríguez-Ortega JÁ, Quiles-Granado J, Franco-López Á. Válvula mitral en paracaídas Complejo de Shone atípico. Cardiocore. 2016;51:83-6.

10. Mamaladze V, Capdeville M, Navia J, Vivacqua A. Parachute-like asymmetric mitral valve associated with mitral valve cleft and atrial septal defect in an adult. J Cardiothorac Vasc Anesth. 2011;25:1106-8.

11. Hakim FA, Kendall CB, Alharthi M, Mancina JC, Tajik JA, Mookadam F. Parachute mitral valve in adults-a systematic overview. Echocardiography. 2010;27:581-6.

12. Kilner PJ. Imaging congenital heart disease in adults. $\mathrm{Br} \mathrm{J}$ Radiol. 2011;84(3:):S258-68

13. Stout KK, Broberg CS, Book WM, Cecchin F, Chen JM, Dimopoulos $\mathrm{K}$, et al. Chronic heart failure in congenital heart disease: a scientific statement from the American Heart Association. Circulation. 2016;133:770-801.

14. Zomer AC, Vaartjes I, van der Velde ET, de Jong HM, Konings TC, Wagenaar LJ, et al. Heart failure admissions in adults with congenital heart disease; risk factors and prognosis. Int J Cardiol. 2013;168:2487-93.

15. Mohan JC, Shukla M, Mohan V, Sethi A. Parachute mitral valve and Pacman deformity of the ventricular septum in a middleaged male. Indian Heart J. 2016;68 Suppl 2:S126-30.

16. Rim Y, McPherson DD, Kim H. Effect of Congenital Anomalies of the Papillary Muscles on Mitral Valve Function. J Med Biol Eng. 2015;35:104-12.

17. Séguéla PE, Houyel L, Acar P. Congenital malformations of the mitral valve. Arch Cardiovasc Dis. 2011;104(8-9):465-79.

18. Quah JX, Hofmeyr L, Haqqani H, Clarke A, Rahman A, Pohlner P, et al. The management of the older adult patient with anomalous left coronary artery from the pulmonary artery syndrome: a presentation of two cases and review of the literature. Congenit Heart Dis. 2014;9:E185-94.

19. Kothari J, Lakhia K, Solanki P, Parmar D, Boraniya H, Patel S. Anomalous origin of the left coronary artery from the pulmonary artery in adulthood: challenges and outcomes. Korean J Thorac Cardiovasc Surg. 2016;49:383-6.

20. Boris JR, Brothers JA. Primary-care management of patients with congenital anomalies of the coronary arteries. Cardiol Young. 2015;25:1540-5.

21. Silverman NH. Echocardiographic presentation of anomalous origin of the left coronary artery from the pulmonary artery. Cardiol Young. 2015;25:1512-23. 